\title{
The tree property at successors of singular cardinals
}

\author{
Menachem Magidor and Saharon Shelah * \\ Institute of Mathematics \\ The Hebrew University, Jerusalem, Israel
}

September 26, 2018

\begin{abstract}
Assuming some large cardinals, a model of ZFC is obtained in which $\aleph_{\omega+1}$ carries no Aronszajn trees. It is also shown that if $\lambda$ is a singular limit of strongly compact cardinals, then $\lambda^{+}$carries no Aronszajn trees.
\end{abstract}

\section{Introduction}

The main results of this paper are (1) that the consistency of "ZFC and $\aleph_{\omega+1}$ carries no Aronszajn trees" follows from the consistency of some large cardinals (a huge cardinal with $\omega$ supercompact cardinals above it), and (2) that if a singular cardinal $\lambda$ is a limit of strongly compact cardinals, then there are no Aronszajn trees of height $\lambda^{+}$. The proof of (2) is in Section 3, and the forcing constructions which prove (1) are given in Sections 4, 5, and 6 . The generalization to higher singular cardinals of both (1) and (2) poses no problem.

Section 2 contains some notations, definitions, and standard constructions.

\section{Preliminaries}

We use the convention concerning forcing by which $p<q$ means that $q$ is more informative than $p$. A forcing poset for us here is a separative partial order $P$, with a least informative point (denoted $\emptyset_{P}$ ) and with no maximal point. (A poset is separative if: Whenever $p \nless q$ then some extension of $q$ in $P$ is incompatible with $p$. There is a canonical way of producing a separative poset from non-separative: Define an equivalence relation $p_{1} \sim p_{2}$ if "any $x$

*Partially sponsored by the Edmund Landau Center for research in Mathematical Analysis, supported by the Minerva Foundation (Germany), p.n. 
is compatible with $p_{1}$ iff it is compatible with $p_{2}$ ". Then, on the equivalence classes, define $\left[p_{1}\right] \leq\left[p_{2}\right]$ if every $x$ compatible with $p_{2}$ is also compatible with $p_{1}$.)

$V^{P}$ denotes the class of all $P$-terms, but when an expression such as " $\mathbf{T} \in$ $V^{P}$ is a tree of hight $\lambda^{+}$is used, we mean that $\emptyset_{P}$ forces this statement.

An Aronszajn tree of height $\lambda^{+}$( $\lambda$ a cardinal) is a tree with $\lambda^{+}$levels, each of cardinality $\leq \lambda$, such that there is no branch of size $\lambda^{+}$. For the question that interests us, it does no harm to assume that all the levels are of size $\lambda$, and it is technically useful to assume that the $\alpha$ th level is always the set $\lambda \times\{\alpha\}$. So if $K$ is a forcing poset and $\mathbf{T} \in V^{K}$ a name of a $\lambda^{+}$-tree, then the underlying universe of $\mathbf{T}$ is in $V$, but, of course, the ordering $<_{\mathbf{T}}$ is obtained by forcing.

Definition 2.1 Let $\mathbf{T}$ be a $K$-name of a $\lambda^{+}$-tree as above, where $\lambda$ and $\lambda^{+}$are cardinals both in $V$ and $V^{K}$, then the pre-tree of $\mathbf{T}$ is the system of relations $\left\langle R_{p} \mid p \in K\right\rangle$ satisfying

$$
a R_{p} b \text { iff } p \Vdash_{K} \quad a<_{\mathbf{T}} b .
$$

So $R_{p}$ is a relation on the universe of $T$, namely on $\lambda \times \lambda^{+}$.

It turns out that the consistency proof for "no Aronszajn trees on $\lambda^{+}$" relies on an investigation of such systems of pre-trees, when $\|K\|<\lambda$; the crucial properties of which are collected in the following definition. The reader who prefers to do so, however, can always think in terms of trees, pre-trees etc. without any harm.

Definition 2.2 Systems: Suppose that $\tau \leq \lambda$ are cardinals, $D \subseteq \lambda^{+}$is unbounded, and $T=\left\langle T_{\alpha} \mid \alpha \in D\right\rangle$ is a sequence such that $T_{\alpha} \subseteq \tau \times\{\alpha\}$, for $\alpha \in D$. Let $I$ be an index set of cardinality $\leq \lambda$, and $R=\left\{R_{i} \mid i \in I\right\}$ a collection of binary relations that are subsets of $\bigcup\left\{T_{\alpha} \times T_{\beta} \mid \alpha<\beta\right.$ in $\left.D\right\}$. Then the pair $\mathcal{S}=(T, R)$ is called a system over $\lambda^{+}$(or a $\lambda^{+}{ }_{-}$system) if the following hold:

1. For every $\alpha<\beta$ in $D$, there are $a \in T_{\alpha}, b \in T_{\beta}$ and $i \in I$ such that $\langle a, b\rangle \in R_{i}$.

2. For every $i \in I$, and $\alpha<\beta<\gamma$ in $D$, if $a \in T_{\alpha}, b \in T_{\beta}, c \in T_{\gamma}$ are such that $\langle a, c\rangle \in R_{i}$ and $\langle b, c\rangle \in R_{i}$, then $\langle a, b\rangle \in R_{i}$.

The set $D$ is called the domain of the system and I its index set. The cardinal $\tau$ is the width, and $\lambda^{+}$the height of the system.

An example of a $\lambda^{+}$-system is any $\lambda^{+}$-tree; in this example $R$ consists of a single relation-the tree ordering. The pre-tree as defined above is more illustrative; the number of relations is the cardinality of the forcing poset.

Strong systems: If condition (1) above is replaced by: "For every $\alpha<\beta$ in $D$, for every $b \in T_{\beta}$, there are $a \in T_{\alpha}$, and $i \in I$ such that $\langle a, b\rangle \in R_{i}$ " (but (2) remains unchanged) then the system $\mathcal{S}$ is called a strong system. 
A pre-tree relative to some forcing poset $K$ is in fact a strong system.

Subsystems: If $\mathcal{S}$ is a system then a subsystem of $\mathcal{S}$ is a system obtained by taking an unbounded subset $D_{0} \subseteq D$, an initial segment $\tau_{0} \leq \tau$, a subset $I_{0} \subseteq I$, and restricting $\mathcal{S}$ to $\left\langle\tau_{0} \times\{\alpha\} \mid \alpha \in D_{0}\right\rangle$ and to the relations with index in $I_{0}$.

By definition a subsystem is a system (though item 2 is inherited automatically from the system, item 1 may not). A subsystem of a strong system may no longer be strong.

Narrow systems: A system $\mathcal{S}$ is said to be $(\rho, \iota)$-narrow if $\tau<\rho$, and $|I|<\iota$. That is, its width is less than $\rho$ and its index set has size $<\iota . A \lambda^{+}$-system is said to be narrow iff it is $(\lambda, \lambda)$-narrow.

Branches: $A$ "branch" of the system is a set $B$ such that for some $i \in I$ for all $a, b \in B\langle a, b\rangle \in R_{i}$ or $\langle b, a\rangle \in R_{i}$.

Thus, a branch of a pre-tree gives a set $B$ forced by a single condition to be linearly ordered.

Pre-systems Suppose that $Q$ is a forcing poset and $\underset{\sim}{\mathcal{S}}=\langle\mathbf{T}, \mathbf{R}\rangle$ is a name in $V^{Q}$ of a system with domain $\lambda^{+}$, width $\lambda_{0} \leq \lambda$, and index set some cardinal $\tau$. Then the pre-system, $\operatorname{Pre}_{Q}(\underset{\sim}{\mathcal{S}})$ is defined as the following $\lambda^{+}$system in $V . \operatorname{Pre}_{Q}(\underset{\sim}{\mathcal{S}})$ has as index set the product $Q \times \tau$, its width remains $\lambda_{0}$, and its relations $R_{q, i}$ are defined for $q \in Q$ and $i \in \tau$ by:

$$
\langle a, b\rangle \in R_{q, i} \text { iff } q \Vdash\langle a, b\rangle \in \mathbf{R}_{i} .
$$

A pre-tree is an example of a pre-system. A pre-system is a system, and it is strong if the system $\mathcal{S}$ is strong in $V^{Q}$ (that is, forced by every condition to be strong). If $P=Q \times K$ is a product and $\underset{\sim}{\mathcal{S}}$ is a $P$-name of a system, then $\operatorname{Pre}_{P}(\underset{\sim}{\mathcal{S}}) \sim \operatorname{Pre}_{Q}\left(\operatorname{Pre}_{K}(\underset{\sim}{\mathcal{S}})\right)$.

\subsection{On collapses}

For a regular cardinal $\kappa$ and an ordinal $\lambda>\kappa, \operatorname{Coll}(\kappa, \lambda)$ is the poset that collapses $\lambda$ to $\kappa$, using functions from ordinals below $\kappa$ and into $\lambda$. The poset $\operatorname{Coll}(\kappa,<\lambda)$ is the product with support of cardinality $<\kappa$ of all collapses of ordinals between $\kappa$ and $\lambda$ (The "Levy" collapse, see Jech, Section 20). Now if $L=\left\langle\lambda_{i}\right| i\langle\omega\rangle$ is an increasing sequence of cardinals, then $C=\operatorname{Coll}(L)$ is the full support iteration of the collapsing posets $\operatorname{Coll}\left(\lambda_{i},<\lambda_{i+1}\right)$. In more details, define by induction on $n<\omega$ posets $P_{n}$ as follows. $P_{1}=\operatorname{Coll}\left(\lambda_{0},<\lambda_{1}\right)$, and

$$
P_{n+1}=P_{n} * \operatorname{Coll}\left(\lambda_{n-1},<\lambda_{n}\right)^{V^{P_{n}}} .
$$


Then $\operatorname{Coll}(L)$ is the full support iteration of the posets $P_{n}$.

A projection from a poset $P$ into $Q$ is an order preserving map $\Pi: P \longrightarrow Q$ such that $\Pi\left(\emptyset_{P}\right)=\emptyset_{Q}$, and if $\Pi(p)=q$ and $q^{\prime}>q$ in $Q$ then for some $p^{\prime}>p$, $\Pi\left(p^{\prime}\right) \geq q^{\prime}$. (Some authors use a different definition!)

If $\Pi: P \longrightarrow Q$ is a projection and $G \subset Q$ is a $V$-generic filter, then $P / G$ is the separative poset defined by taking $\Pi^{-1} G$ and turning it into a separative poset. Then $P$ is isomorphic to a dense subset of the iteration $Q *(P / G)$ : the isomorphism is the map taking $p \in P$ to $(\Pi(p),[p])$.

Lemma 2.1 Suppose that $Q$ is a $\lambda$-complete poset (any increasing sequence of length $<\lambda$ has a least upper bound). Let $\mu$ be the cardinality of $Q$. Then there is a projection $\Pi: \operatorname{Coll}(\lambda, \mu) \longrightarrow Q$ such that whenever $G \subset Q$ is $V$ generic, then the quotient poset $\operatorname{Coll}(\lambda, \mu) / G$ is $\lambda$-complete. In fact, the projection is $\lambda$-continuous: If $f=\bigcup_{i<\lambda_{0}} f_{i}$ is the supremum of an increasing sequence of lenght $\lambda_{0}<\lambda$ of conditions in $\operatorname{Coll}(\lambda, \mu)$ then $\Pi(f)$ is the supremum of $\left\{\Pi\left(f_{i}\right) \mid\right.$ $\left.i<\lambda_{0}\right\}$.

Proof. Let $Q=\left\{q_{i} \mid i<\mu\right\}$ be an enumeration of $Q$. Any condition in $\operatorname{Coll}(\lambda, \mu)$ is a function $f: \alpha \longrightarrow \mu$, where $\alpha<\lambda$, and we define $\Pi(f)$ as follows. Define by induction an increasing sequence $\langle a(\xi) \mid \xi \leq \alpha\rangle$ by requiring that (1) $a(0)$ is the minimum of $Q(2)$ at limit stages $\delta \leq \alpha, a(\delta)$ is the least upper bound of $\langle a(\xi) \mid \xi<\delta\rangle$, and (3) if $q_{f(\xi)}$ extends $a(\xi)$ in $Q$, then $a(\xi+1)=q_{f(\xi)}$, and otherwise $a(\xi+1)=a(\xi)$. Finally, $\Pi(f)=a(\alpha)$. It is easy to see that $\Pi$ is a $\lambda$-continuous projection.

Remark first that if $G \subset Q$ is $V$-generic, then $\Pi^{-1} G$ is already separative. To prove the $\lambda$-closure of the quotient, suppose that $q \Vdash\left\langle\tau_{i} \mid i<\lambda_{0}<\lambda\right\rangle$ is an increasing $Q$-sequence in $\operatorname{Coll}(\lambda, \mu) / G=\Pi^{-1} G$. We will find an extension of $q$ that forces a least upper bound to this sequence. Define by induction on $i<\lambda_{0}$ an increasing sequence $\left\langle q_{i} \mid i<\lambda_{0}\right\rangle$ beginning with $q_{0}=q$, such that for every $i$, for some $f_{i} \in \operatorname{Coll}(\lambda, \mu), q_{i+1} \Vdash \tau_{i}=f_{i}$. Let $q^{\prime}$ be an upper bound in $Q$ to this sequence. Now $f_{i} \subset f_{j}$ for $i<j$, and $f=\bigcup_{i<\lambda_{0}} f_{i}$ is a condition. Since $Q$ is separative, $\Pi\left(f_{i}\right) \leq_{Q} q_{i+1}$ follows from the fact that $q_{i+1} \Vdash \Pi\left(f_{i}\right) \in G$. The continuity of $\Pi$ implies that $\Pi(f)$ is the least upper bound of all the conditions $\Pi\left(f_{i}\right)$, and hence $\Pi(f) \leq q^{\prime}$. That is $q^{\prime}$ forces that $f$ is in $\operatorname{Coll}(\lambda, \mu) / G$.

A similar lemma holds for $\operatorname{Coll}(\lambda,<\kappa)$ if the cardinality of $Q$ is less than $\kappa$ (and is $\lambda$-complete), but we need to apply such a lemma in a slightly more complex situation. Suppose that:

1. $\lambda<\lambda_{1} \leq \mu<\kappa$ are regular cardinals. $Q=\operatorname{Coll}\left(\lambda,<\lambda_{1}\right)$, and the projection $\Pi_{1}: \operatorname{Coll}(\lambda,<\kappa) \longrightarrow Q$ is the obvious restriction projection.

2. $P=Q * R$ is a two stage iteration where $R$ is a name in $V^{Q}$ such that $R$ is forced to be $\lambda$-closed (by every condition). The projection of $P$ on $Q$ is denoted $\Gamma$ (so $\Gamma(q, \tau)=q)$.

Suppose that the cardinality of $P$ is $\mu$. 
Lemma 2.2 Under the conditions set above on $P, Q$, and $R$, there is a projection $\Pi: \operatorname{Coll}(\lambda,<\kappa) \longrightarrow P$ such that $\Gamma \circ \Pi=\Pi_{1}$, and such that whenever $G \subseteq P$ is $V$-generic, then the quotient poset $\operatorname{Coll}(\lambda,<\kappa) / G$ is $\lambda$-closed.

Proof. Set an enumeration $\left\{\tau_{i} \mid i \in \mu\right\}$ of all the terms in $V^{Q}$ that are forced by every condition to be in $R$, and where two names are identified if every condition forces them to be equal. Given any condition $q \in \operatorname{Coll}(\lambda,<\kappa)$, let $q_{1}=\Pi_{1}(q)$, and $f=q\lceil\{\mu\}$ be the component of $q$ that collapses $\mu$. Then $\Pi(q)=\left(q_{1}, \tau\right) \in Q * R$ is defined by the following procedure. Suppose that the domain of $f$ is $\alpha<\mu$, and define an increasing sequence $\left\langle\eta_{i} \mid i \leq \alpha\right\rangle$ of terms, by induction on $i$ as follows:

1. $\eta_{0}$ is an assumed empty condition in $R$ (least informative). $\eta_{i+1}$ is $\tau_{f(i)}$ if every condition in $Q$ forces that $\tau_{f(i)}$ extends $\eta_{i}$, and $\eta_{i+1}=\eta_{i}$ otherwise.

2. If $\delta \leq \alpha$ is a limit ordinal and all the terms $\eta_{i}$ for $i<\delta$ have been defined such that for $i<j<\delta, \eta_{i}<_{R} \eta_{j}$ is forced by every condition in $Q$, then $\eta_{\delta}$ is defined as (the name of) the least upper bound of this increasing sequence.

Finally, the projection is defined by setting $\tau=\eta_{\alpha}$. We leave it to the reader to verify that $\Pi$ is indeed a projection as required, and in particular that if $G$ is generic over $P$ then the quotient $\operatorname{Coll}(\lambda,<\kappa) / G$ is $\lambda$-closed.

\subsection{On embeddings and ultrapowers}

The dual characterization of supercompact cardinals is probably known to the reader: If $\mu>\kappa$ then $\kappa$ is $\mu$-supercompact if $\kappa$ is the critical point (first fixed point) of an elementary embedding $j: V \longrightarrow M$ of the universe $V$ into a transitive inner model $M$ such that $\mu<j(\kappa)$ and $M^{\mu} \subset M$. An alternative, more tangible, definition is that $P_{\kappa}(\mu)=\{X|X \subseteq \mu| X \mid,<\kappa\}$ carries a non-principal, fine, $\kappa$-complete, normal ultrafilter. (See Jech [] Chapter 6, or Solovay $\mathrm{xxxxx}$ ).

We say that $\kappa$ is huge if $\kappa$ is the critical point of an elementary embedding $j: V \longrightarrow M$ into a transitive substructure $M$ such that $M^{j(\kappa)} \subseteq M$. If the stronger demand, $M^{j(j(\kappa))} \subseteq M$, holds then we say that $\kappa$ is 2-huge. For our consistency result we need a cardinal that is slightly stronger than huge, but not quite 2-huge. Its definition is given by the following lemma on the equivalence between two characterizations, which we quote without proof (the proof is quite standard; see for example SRK).

If $\mu>\tau$ are cardinals, define $P_{\mu}^{\tau}=\{X \subseteq \mu \mid$ order-type of $X$ is $\tau\}$. If $\mathcal{U} \subset \mathcal{P}\left(P_{\mu}^{\tau}\right)$ is a non-principal ultrafilter, then $\mathcal{U}$ is said to be:

1. $\kappa$-complete if the intersection of fewer than $\kappa$ sets in $\mathcal{U}$ is again in $\mathcal{U}$. 
2. normal if any choice function $f$ is constant on a set in $\mathcal{U}$. ( $f$ is a choice function if $f(A) \in A$ for every $A \in P_{\mu}^{\tau}$. $f$ is constant on $X \in \mathcal{U}$ if for some $\gamma \in \mu, f(A)=\gamma$ for all $A \in X$.)

3. fine if $\forall \alpha<\mu\{A \mid \alpha \in A\} \in \mathcal{U}$.

4. $\kappa$-small below $\tau$ if $\left\{A \in P_{\mu}^{\tau}|| A \cap \tau \mid<\kappa\right\} \in \mathcal{U}$.

Lemma 2.3 1. If $j: V \longrightarrow M$ is an elementary embedding into a transitive structure $M$, with critical point $\kappa$, and $\tau$ is such that $\kappa<\tau<j(\kappa)<$ $j(\tau)=\mu$ and $M^{\mu} \subseteq M$, if $\mathcal{U} \subset \mathcal{P}\left(P_{\mu}^{\tau}\right)$ is defined by

$$
A \in \mathcal{U} \quad \text { iff }\left(j^{\prime \prime} \tau\right) \in j(A),
$$

then $\mathcal{U}$ is a non-principal, $\kappa$-complete, normal, fine, and $\kappa$-small below $\tau$ ultrafilter on $P_{\mu}^{\tau}$.

2. If, on the other hand, $\kappa<\tau<\mu$ and $\mathcal{U}$ are such that $\mathcal{U} \subset \mathcal{P}\left(P_{\mu}^{\tau}\right)$ is an ultrafilter that satisfy the five properties above, and if the ultrapower $V^{P_{\mu}^{\tau}} / \mathcal{U}$ is created, then it is well-founded and the ensuing elementary embedding, $i: V \longrightarrow N$, is such that $\kappa$ is the critical point of $i, \tau<i(\kappa)$, $\mu=i(\tau)$ and $N^{i(\tau)} \subset N$.

\subsection{The exact assumptions}

For the consistency proof of "no Aronszajn trees on $\aleph_{\omega+1}$ " we need the following:

A cardinal $\kappa$ and an increasing sequence of cardinals $L=\left\langle\lambda_{i} \mid i<\omega\right\rangle$ with $\lambda_{0}>\kappa$ such that:

$A_{1}$ : Let $\lambda=\sup \left\{\lambda_{i} \mid i<\omega\right\}$ and $\mu=\lambda^{+}$, then each $\lambda_{i}$, for $i>0$, is $\mu$-supercompact.

$A_{2}$ : If $P=\operatorname{Coll}(L)$, then in $V_{1}=V^{P} \kappa$ is $\tau$-huge for $\tau=\kappa^{+(\omega+1)}$ and an embedding $j: V_{1} \longrightarrow M_{1}$ (as in Lemma 2.3) such that $j(\tau)=\mu$.

This requirement of a "potentially" huge with $\omega$ supercompacts above it, is somewhat technical, but it may be obtained with the following more familiar assumptions:

A cardinal $\kappa$ and an increasing sequence $\left\langle\lambda_{i} \mid i<\omega\right\rangle$ such that:

$B_{1}:$ For $\lambda=\sup \left\{\lambda_{i} \mid i<\omega\right\}$ and $\mu=\lambda^{+}$, each $\lambda_{i}, i>0$, is $\mu-$ supercompact.

$B_{2}: \kappa$ is the critical point of an embeding $j: V \longrightarrow M$ where $j(\kappa)=\lambda_{0}$ and $M^{\mu} \subseteq M$. 
Our aim in this subsection is to prove that if cardinals $\kappa$ and $\left\langle\lambda_{i} \mid i<\omega\right\rangle$ satisfy $B_{1}$ and $B_{2}$, then there is a generic extension in which cardinals that satisfy $A_{1}$ and $A_{2}$ can be found.

Let $\rho$ be a cardinal, and $L=\left\langle\lambda_{i} \mid i<\omega\right\rangle$ be an increasing sequence with limit $\lambda$ of cardinals such that, $\lambda_{0}=\rho$ and for $i>0, \lambda_{i}$ is $\lambda^{+}$-supercompact. Than $L$ is called "the minimal supercompact sequence above $\rho$ " if $\lambda$ is the least cardinal above $\rho$ such that the interval $(\rho, \lambda)$ possesses an $\omega$-sequence of $\lambda^{+}$supercompact cardinals, $\lambda_{0}=\rho$, and each $\lambda_{i+1}$ is the first $\lambda^{+}$-supercompact cardinal above $\lambda_{i}$.

Fix a function $g$ such that, for every $\rho, g(\rho)=\left\langle\lambda_{i} \mid i<\omega\right\rangle$ is such that $\lambda_{0}=\rho$ and $\left\langle\lambda_{i} \mid i<\omega\right\rangle$ is the minimal supercompact sequence above $\lambda_{0}$ (if it exists, and $g$ is undefined otherwise).

Looking at $j^{2}$, it is not difficult to see that if $\kappa$ is huge, then it is $j(\kappa)$ supercompact, not only in $V$ but in $M$ as well (use the combinatorial characterization of supercompactness). Hence, as high as we wish below $\kappa$, there are cardinals that are $\kappa$-supercompact. So, for every $\rho<\kappa, g(\rho)<\kappa$ (is defined), and thus, for every $\rho<j(\kappa), g(\rho)<j(\kappa)$ as well.

Let $\kappa, \mu$, and $\left\langle\lambda_{i} \mid i<\omega\right\rangle$ be as in $B_{1}$ and $B_{2}$. So $\kappa$ is the critical point of an embedding $j: V \longrightarrow M$, where $M^{\mu} \subseteq M$, and (by taking the minimal sequence above $j(\kappa)$ ) we may assume that

$$
g(j(\kappa))=\left\langle\lambda_{i} \mid i<\omega\right\rangle=L .
$$

Thus, if $L_{0}$ is the minimal supercompact sequence above $\kappa$, and $\tau=\left(\sup \left(L_{0}\right)\right)^{+}$, then $j\left(L_{0}\right)=L$, and $j(\tau)=\mu$. $\kappa$ is thus $\tau$-huge (as in Lemma 2.3).

For every ordinal $\alpha \leq j(\kappa)$ a cardinal $\rho_{0}(\alpha)$ and an iteration $P_{\alpha}$ of length $\alpha$ with Easton support, is defined by induction. Then, finally, $P_{j(\kappa)}$ is the required poset which gives an extension where $\kappa$ and $L$ satisfy $A_{1}$ and $A_{2}$.

1. $P_{0}$ is the trivial poset and $\rho_{0}(0)=\aleph_{1}$.

2. For limit $\alpha, P_{\alpha}$ consists of all partial functions $f$ defined on $\alpha$ such that $f \uparrow$ $\gamma \in P_{\gamma}$ for all $\gamma<\alpha$, and $f$ has the Easton support property: $\operatorname{dom}(f) \cap \gamma$ is bounded below $\gamma$ for every inaccessible $\gamma$. The cardinal $\rho_{0}(\alpha)$ is the first inaccessible cardinal above all the $\rho_{0}(\gamma)$ 's, $\gamma<\alpha$.

3. If $P_{\alpha}$ and $\rho_{0}(\alpha)$ are defined, then $P_{\alpha+1}=P_{\alpha} * \operatorname{Coll}\left(L^{\alpha}\right)$ for $L^{\alpha}=g\left(\rho_{0}(\alpha)\right)$. The first inaccessible above the cardinals in $L$ is $\rho_{0}(\alpha+1)$.

Standard arguments prove that for Mahlo $\gamma$ 's that are closed under the function $\alpha \mapsto \sup (g(\alpha)), P_{\gamma}$ satisfies the $\gamma$-c.c. Also, for any $\alpha<\beta, P_{\beta}$ can be decomposed as $P_{\alpha} * R$, where $R$ is defined in $V^{P_{\alpha}}$ as an Easton support iteration of collapses determined by the same function $g$, but beginning with $\rho_{0}(\alpha)$. (Supercompact cardinals remain supercompact in any generic extension done via a poset of smaller size; see LLL.) 
Set $P=P_{j(\kappa)}$. We will show in $V^{P}$ that $\kappa$ and $L=g(j(\kappa))$ satisfy the properties $A_{1}$ and $A_{2}$. Recall that $\mu=\lambda^{+}$where $\lambda=\sup (L)$. In $V$, we have an elementary embedding $j: V \longrightarrow M$ into a transitive inner model $M$ such that $M^{\mu} \subseteq M$. Again, the argument that small forcing will not destroy supercompacness can show that the sumpercompact cardinals in $L$ remain $\mu$-supercompact in $V^{P}$; that is, $A_{1}$ is easy. We promised to prove that in $\left(V^{P}\right)^{\operatorname{Coll}(L)}, \kappa$ is $\tau$-huge for $\tau=\kappa^{+(\omega+1)}$, but in fact we will find a condition in $P * \operatorname{Coll}(L)$ and show that extensions through this condition satisfy this requirement. The argument is fairly standard, but we repeat it for completeness' sake.

Observe that

$$
j\left(P_{\kappa+1}\right)=\left[P_{j(\kappa+1)}\right]^{M},
$$

but the closure of $M$ under $\mu$ sequences implies that interpreting this iteration in $V$ or in $M$ results in the same poset $Q$. So

$$
Q=j\left(P_{\kappa+1}\right)=P_{j(\kappa)+1}=P_{j(\kappa)} * \operatorname{Coll}(L) .
$$

We will find a condition $q \in Q$ that forces $\kappa$ to be $\tau$-huge (as in Lemma 2.3). In fact, the following suffices:

Lemma 2.4 There is a condition $q \in Q$ such that if $K$ is a $V$-generic filter over $Q$ containing $q$, then the collection $\{j(p) \mid p \in K\}$ has an upper bound in $j(Q) / K$.

The meaning and proof of this lemma are clarified by the following: Decompose

$$
Q=P_{\kappa+1} * R
$$

where $R$, the remainder, is an Easton support iteration, starting above $\operatorname{Coll}(g(\kappa))$, of collapses guided by $g$, going up to $j(\kappa)+1$. Now apply $j$ to get

$$
j(Q)=\left[P_{j(\kappa)+1} * j(R)\right]^{M}=[Q * j(R)]^{M} .
$$

So $Q$ is a factor of $j(Q)$, and $j(Q) / K$ can be formed in $M[K]$. The lemma claims first that each $j(p)$, for $p \in K$, is in $j(Q) / K$, and then that this collection has an upper bound.

Since $2^{\lambda}=\mu$, the cardinality of $Q$ is $\mu$. It follows that in $V[K](M[K])^{\mu} \subseteq$ $M[K]$. Certainly, $j(Q) / K$ is $\mu^{+}$-closed in $M[K]$ (in fact it is $\tau_{0}$-closed, where $\tau_{0}$ is the first inaccessible in $M$ above $\lambda$ ). Hence $j(Q) / K$ is $\mu^{+}$-closed in $V[K]$. So, to prove the lemma, we only need to choose $q \in Q$ which forces that

$$
j(p) \in j(Q) / K
$$

for $p \in K$.

Analyzing (11), we write $p \in Q$ as $p=\left\langle p_{0}, t, r\right\rangle$ where $p_{0} \in P_{\kappa}, t$ is a name, force to be in $\operatorname{Coll}(L)$, and $r$ is forced to be in $R$. Then $j(p)=\left\langle p_{0}, j(t), j(r)\right\rangle$ 
(because $j\left(p_{0}\right)=p_{0}$ by the Easton condition). Now $j(p) \in j(Q) / K$ iff the projection of $j(p)$ on $Q$, namely $\left\langle p_{0}, j(t)\right\rangle$, is in $K$.

The definition of $q$ can now be given, Define $q \in P_{j(\kappa)+1}$ as $\langle\emptyset, \sigma\rangle$, where $\sigma \in V^{P_{j(\kappa)}}$ is forced to be in $\operatorname{Coll}(L)$. It is easier to describe the interpretation of $\sigma$ in $V[H]$, where $H \subset P_{j(\kappa)}$ is $V$-generic. Well, look at all conditions $p \in$ $P_{\kappa+1} \cap H$ (there are $<j(\kappa)$ of them); write each such $p$ as $p=\left\langle p_{0}, t\right\rangle$; interpret $t$ as a condition in $\operatorname{Coll}(L)$, and take the supremum in $\operatorname{Coll}(L)$ of all of these condition. This proves the lemma and we now see how the result follows.

Lemma 2.5 Assuming $q$ is as in Lemma 2.4, $q \Vdash_{Q} \kappa$ is $\tau$-huge.2.3.

Proof. Let $K \subseteq Q$ be a $V$-generic filter containing $q$. Work in $V[K]$ and let $s_{0} \in j(Q) / K$ be an upper bound of $\{j(p) \mid p \in K\}$. We are going to define in $V[K]$ an ultrafilter $\mathcal{U}$ over $P_{\mu}^{\tau}$ that satisfy the properties of Lemma 2.3. For this, we fix $\left\langle A_{\xi} \mid \xi \in \mu^{+}\right\rangle$, an enumeration af all subsets of $P_{\mu}^{\tau}$, and plan to decide inductively whether $A_{\xi} \in \mathcal{U}$ or not. Construct by induction an increasing sequence $\left\langle s_{\xi} \mid \xi<\mu^{+}\right\rangle$of conditions in $j(Q) / K$ as follows:

1. At limit stages, $\delta<\mu^{+}$, use the $\mu^{+}$-completeness of $j(Q) / K$ to find an upper bound to $\left\langle s_{\xi} \mid \xi<\delta\right\rangle$.

2. If $s_{\xi}$ is defined, pick for $A_{\xi}$ a name $a_{\xi}$ such that $a_{\xi}[K]$, the interpretation of $a_{\xi}$ in $V[K]$, is $A_{\xi}$. Then $j\left(a_{\xi}\right) \in M^{j(Q)}$, and we find an extension $s_{\xi+1}$ of $s_{\xi}$ that decides whether $\left(j^{\prime \prime} \tau\right) \in j\left(a_{\xi}\right)$ or not. If the decision is positive, then put $A_{\xi} \in \mathcal{U}$, and otherwise not. Two comments are in order for this definition to make sense:

(a) First, $j\left(a_{\xi}\right)$ is not a name in $j(Q) / K$-forcing, but in $j(Q)$. Yet, from $M[K]$ any generic extension via $j(Q) / K$ takes us into a universe that is also a $j(Q)$ generic extension of $M$, and it is as such that we ask about the interpretation of $j\left(a_{\xi}\right)$.

(b) Apparently, this definition depends on a particular choice of a name for $A_{\xi}$, but in fact if $a_{\xi}^{\prime}$ is another name, then the same answer is obtained. The point of the argument is that some condition $p$ in $K$ forces $a_{\xi}=a_{\xi}^{\prime}$, and hence $\left[j(p) \Vdash_{j(Q)} j\left(a_{\xi}\right)=j\left(a_{\xi}^{\prime}\right)\right]^{M}$. But since $j(p) \in j(Q) / K$ is extended by $s_{0}$, it can be seen that the answer to the $\xi$ 's question does not depend on the particular choice of the name.

We leave it to the reader to prove that $\mathcal{U}$ thus defined satisfies the required properties of Lemma 2.3. For example, let us prove that $\mathcal{U}$ is $\kappa$-small below $\tau=\kappa^{+(\omega+1)}$ in $V[K]$. For some $\xi \in \mu^{+}, A_{\xi}=\left\{A \in P_{\mu}^{\tau}|| A \cap \tau \mid<\kappa\right\}$, and a name $a_{\xi}$ for $A_{\xi}$ was chosen and an extension $s_{\xi+1}$ deciding whether $j^{\prime \prime} \mu \in j\left(a_{\xi}\right)$ was thought after. But some condition $p \in K$ forces $a_{\xi}=\left\{A \in P_{\mu}^{\tau}|| A \cap \tau \mid<\kappa\right\}$, and hence $j(p)$, and decidedly $s_{0}$, forces $j\left(a_{\xi}\right)=\left\{A \in P_{j(\mu)}^{j(\tau)}|| A \cap j(\tau) \mid<j(\kappa)\right\}$. 
Now $j^{\prime \prime} \mu=A$ has order-type $\mu=j(\tau)$, and $A \cap j(\tau)=j^{\prime \prime} \tau$ has cardinality $\tau<j(\kappa)$.

\section{There are no Aronszajn trees on successors of singular limits of compact cardinals}

The paper really begins here, with the following theorem of ZFC.

Theorem 3.1 If $\lambda$ is singular and a limit of strongly compact cardinals, then there are no $\lambda^{+}$-Aronszajn trees.

Proof. For notational simplicity, assume that $\operatorname{cf}(\lambda)=\omega$. Let $\left\langle\lambda_{i} \mid i<\omega\right\rangle$ be an increasing $\omega$-sequence of strongly compact cardinals with limit $\lambda$. (Recall that a cardinal $\kappa$ is strongly compact if every $\kappa$-complete filter can be extended to a $\kappa$-complete ultrafilter.) Let $T$ be a tree of height $\lambda^{+}$and levels of size $\leq \lambda$ (a $\lambda^{+}$-tree), and we will find a $\lambda^{+}$branch in $T$. We may assume that $T_{\alpha}$, the $\alpha$ th level of $T$, is the set $\lambda \times\{\alpha\}$. Accordingly, we define $T_{\alpha, n}=\lambda_{n} \times\{\alpha\}$, so that $T_{\alpha}=\bigcup_{n<\omega} T_{\alpha, n}$. The proof for the existence of the branch is divided into two steps:

Step one: There is an unbounded $D \subseteq \lambda^{+}$and a fixed $n \in \omega$ such that whenever $\alpha<\beta$ are both in $D$, then, for some $a \in T_{\alpha, n}$ and $b \in T_{\beta, n}, a<_{T} b$. We call an unbounded set $D$ and a collection $\left\langle T_{\alpha, n} \mid \alpha \in D\right\rangle$ as above a spine of $T$. Thus the the first part of the proof provides a spine for every $\lambda^{+}$tree.

Indeed, using the fact that $\lambda_{0}$ is strongly compact, extend the co-bounded subsets of $T$ (that is, those subsets whose complement has cardinality $\leq \lambda$ ) to a countably complete, uniform ultrafilter, $u$, over $T$. Given $\alpha \in \lambda^{+}$ (considered as a level of $T$ ) define $n_{\alpha} \in \omega$ by the following procedure: For every $x \in T$ of level $>\alpha$, let $r_{x}^{\alpha} \in T_{\alpha}$ be such that $r_{x}^{\alpha}<_{T} x$, and set $n=n_{x}$ to be (the least) such that $r_{x}^{\alpha} \in T_{\alpha, n}$. Since the set $T \backslash(T\lceil\alpha+1)$ is in $u$, it follows from the $\aleph_{1}$-completeness of $u$ that for some $n=n_{\alpha},\{x \in T \mid$ $\left.n_{x} \leq n\right\}=X_{\alpha} \in u$.

Now there is an unbounded $D \subseteq \lambda^{+}$and a fixed $n$ such that $n=n_{\alpha}$ for $\alpha \in D$. If we take any two ordinals $\alpha<\beta$ in $D$, then the intersction $X_{\alpha} \cap X_{\beta}$ is in $u$, and any $x$ in this intersection is such that $a=r_{x}^{\alpha}$ and $b=r_{x}^{\beta}$ are comparable (being both below $x$ ), and in the $n$th part, as required.

Step two: Every spine has a cofinal branch. Suppose that $D$ and $n$ define a spine of $T$ as above. That is, assume $\left\{T_{\alpha, n} \mid \alpha \in D\right\}$, where $D \subseteq \lambda^{+}$ is unbounded, is a collection such that for every $\alpha<\beta$ in $D$ there are $a \in T_{\alpha, n}, b \in T_{\beta, n}$ such that $a<_{T} b$. Find a $\lambda_{n+1}$-complete ultrafilter, $v$, 
over $\lambda^{+}$containing $D$ and the co-bounded subsets. Fix any $\alpha \in D$. For every $\beta>\alpha$ in $D$ find $a(\beta) \in T_{\alpha, n}$ and $b(\beta) \in T_{\beta, n}$ such that $a(\beta)<_{T} b(\beta)$. Use the completeness of $v$, and the fact that the cardinality of each level of the spine is only $\lambda_{n}$, to find $a_{\alpha} \in T_{\alpha, n}$ and $\xi_{\alpha} \in \lambda_{n}$ such that for a set of $\beta$ 's in $v, a_{\alpha}=a(\beta)$ and $b(\beta)=\left\langle\xi_{\alpha}, \beta\right\rangle$ (which is the $\xi_{\alpha}$-th element of $\left.T_{\beta, n}=\lambda_{n} \times\{\beta\}\right)$. For an unbounded $D^{\prime} \subseteq D$ the ordinal $\xi_{\alpha}$ has the fixed value $\xi$ for $\alpha \in D^{\prime}$. Now the collection $\left\{a_{\alpha} \mid \alpha \in D^{\prime}\right\}$ is a branch of $T$.

In a very direct way, one can generalize this to find that if $\lambda$ is singular and a limit of strongly compact cardinals as above, then any strong system over $\lambda^{+}$ with index-set of size $<\lambda$ has a branch of size $\lambda^{+}$.

It may be illuminating to remark that the structure of this theorem resembles somewhat the structure of the consistency proof for $\aleph_{\omega+1}$. Assuming that $\lambda$ is a singular limit of strongly compacts, we have proven in Step one that any $\lambda^{+}$-tree (viewed as a strong system) posseses a narrow subsystem. Then, in Step two, it was shown in fact that every narrow system (with fewer than $\lambda$ relations) has a $\lambda^{+}$branch. Now, when proving the consistency theorem (assuming some large cardinals), we will find in the first step a generic extension such that the pre-system of every strong system on $\aleph_{\omega+1}$, with countably many relations, has a narrow subsystem (that is, one of width $\aleph_{n}$ ) in the ground model (Theorem ). So, if in the ground model every narrow system has a cofinal branch, then we are done. Unfortunately, we only know how to arrange the "potential branching property" in the ground model: Every narrow system has a branch in some sufficiently closed generic extension. It turns out that this is sufficient, because of a "preservation theorem" (Theorem 5.2).

\section{The narrowing property}

Definition 4.1 Let $\mu>\chi$ be two cardinals, and $\mu=\lambda^{+}$. A poset $Q$ has the narrow pre-system property for $(\mu, \chi)$ if whenever $\mathcal{S}$ is a $Q$-name of a strong $\mu$-system, with $\leq \chi$ relations, then $\operatorname{Pre}_{Q}(\mathcal{S})$ has a narrow subsystem.

Theorem 4.1 Suppose that $\kappa$ is $\kappa^{+(\omega+1)}$-huge. That is, $\kappa$ is the critical point of an elementary embedding $j: V \rightarrow M$, where $M$ is a transitive class such that $M^{\mu} \subseteq M$, for $\mu=\lambda^{+}=j(\kappa)^{+(\omega+1)}$. Let $Q=\operatorname{Coll}\left(\kappa^{+(\omega+1)},<j(\kappa)\right)$. Then $Q$ has the narrow pre-system property for $\left(\mu, \kappa^{+\omega}\right)$.

Proof. Suppose $\mathcal{S}_{0}$ is forced by every condition in $Q$ to be a strong system on $\mu$, and $\kappa^{+\omega}$ is its index set. Let $\mathcal{S}_{1}=\operatorname{Pre}_{Q}\left(\mathcal{S}_{0}\right)$ be its pre-system; we must find a narrow subsystem of $\mathcal{S}_{1}$ (that is, one of width and index set $<\lambda$ ). The $\alpha$ th level of $\mathcal{S}_{0}$ (and $\left.\mathcal{S}_{1}\right)$ is $\lambda \times\{\alpha\}$, and we denote it by $\left(S_{0}\right)_{\alpha}$. The $n$th part of this level, $j(\kappa)^{+n}$ is denoted $\left(S_{0}\right)_{\alpha, n}$.

Observe that $j(Q)$ is $\mu$-closed in $M$ since $Q$ is $\kappa^{+(\omega+1)}$-closed and $\mu=$ $j\left(\kappa^{+(\omega+1)}\right)$. In fact, $j(Q)$ is $\mu$-closed in $V$ since $M$ is sufficiently closed. 
$j\left(\mathcal{S}_{0}\right)$ is in $V^{j(Q)}$ a strong $j(\mu)$-system with relations indexed by $j(\kappa)^{+\omega}=\lambda$. The $\alpha$ th level of $j\left(\mathcal{S}_{0}\right)$ is $j(\lambda) \times\{\alpha\}$. It is more convenient to denote this level by $\left(j S_{0}\right)_{\alpha}$. Similarly, the $n$th part of this level is denoted $\left(j S_{0}\right)_{\alpha, n}\left(=j(\kappa)^{+n} \times\{\alpha\}\right)$.

It follows from the closure of $M$ under $\mu$-sequences that $j^{\prime \prime} \mu$ is a bounded subset of $j(\mu)$ in $M$, and we let $\beta^{*}<j(\mu)$ be a bound of $j^{\prime \prime} \mu$. Let $b^{*}$ be any fixed ordinal in $j(\lambda) \times\left\{\beta^{*}\right\}$ (so $b^{*}$ is any node of level $\beta^{*}$ in $j\left(\mathcal{S}_{0}\right)$ ).

Inductively, define in $M-\mathrm{a} j(Q)$ increasing sequence of conditions $\left\{s_{\alpha} \mid\right.$ $\alpha<\mu\}$, starting with any condition, as follows:

1. At limit stages, the $\mu$-closure of $j(Q)$ is used to find an upper bound to the sequence of length $<\mu$ so far constructed.

2. If $s_{\alpha}$ is defined, then $s_{\alpha+1}$ is defined as follows: Since $j\left(\mathcal{S}_{0}\right)$ is forced to be a strong system, there exist $a \in\left(j S_{0}\right)_{j(\alpha)}$ and $\zeta<\lambda$ such that $\left\langle a, b^{*}\right\rangle$ is forced by some extension of $s_{\alpha}$ to stand in the $\zeta$ th relation of $j\left(\mathcal{S}_{0}\right)$. So we pick $s_{\alpha+1}$, extending $s_{\alpha}, a_{\alpha} \in\left(j S_{0}\right)_{j(\alpha)}$, and $\zeta_{\alpha}$ such that

$$
s_{\alpha+1} \Vdash_{j(Q)}\left\langle a_{\alpha}, b^{*}\right\rangle \text { stands in the } \zeta_{\alpha} \text { th relation. }
$$

Since $\lambda<\mu$ there is a fixed $\zeta^{0}<\lambda$, and $n \in \omega$, such that for some unbounded set $D \subseteq \mu, \zeta_{\alpha}=\zeta^{0}$ and $a_{\alpha} \in\left(j S_{0}\right)_{\alpha, n}$ for all $\alpha \in D$.

Now the pre-system $\mathcal{S}_{1}$ has width $\lambda$ and relations indexed by $Q \times \kappa^{+\omega}$. We claim that the narrow substructure of $\mathcal{S}_{1}$ defined by $\left\{\left(S_{0}\right)_{\alpha, n} \mid \alpha \in D\right\}$ is a system, thereby proving the theorem. If this is not the case, then for some $\alpha_{1}<$ $\alpha_{2}$ in $D$, "there are no $a_{1} \in\left(S_{0}\right)_{\alpha_{1}, n}$ and $a_{2} \in\left(S_{0}\right)_{\alpha_{2}, n}$ such that $\left\langle a_{1}, a_{2}\right\rangle$ stands in a relation of $\mathcal{S}_{1}$ ", or specifically, "there are no $a_{1} \in\left(S_{0}\right)_{\alpha_{1}, n}, a_{2} \in\left(S_{0}\right)_{\alpha_{2}, n}$, $q \in Q$, and $\zeta \in \kappa^{+\omega}$ such that $q \Vdash_{Q}\left\langle a_{1}, a_{2}\right\rangle$ stand in the $\zeta$ th relation." But then, applying $j$ to this statement we get a contradiction to: $s_{\alpha_{2}+1} \Vdash$ $\left\langle a_{\alpha_{1}}, b^{*}\right\rangle,\left\langle a_{\alpha_{2}}, b^{*}\right\rangle$ and hence $\left\langle a_{\alpha_{1}}, a_{\alpha_{1}}\right\rangle$ as well stand in the $\zeta^{0}$ th relation.

Corollary 4.2 Let $\kappa$ be $\kappa^{+(\omega+1)}$-huge (as in Lemma 2.3). Then it is possible to collapse $\kappa^{+(\omega+1)}$ to be $\aleph_{\omega+1}$ with a forcing poset that has the narrow pre-system property for $\left(j(\kappa)^{+(\omega+1)}, \omega\right)$. In other words, there is a forcing poset $P$ such that

1. $j(\kappa)^{+(\omega+1)}$ becomes $\aleph_{\omega+1}$ in $V^{P}$, and

2. the pre-system $P_{P}$ of every strong system on $j(\kappa)^{+(\omega+1)}$ with countably many relations in $V^{P}$ has a narrow subsystem.

Proof. The desired poset is simply the collapse of $j(\kappa)$ to become $\aleph_{2}$, but not in the most direct way. It is rather the product of two collapses that works: the collapse of $\kappa^{+\omega}$ to $\aleph_{0}$, and the one that makes $j(\kappa)$ the double successor of $\kappa^{+\omega}$ (both posets are defined in $V$ ). Let $Q=\operatorname{Coll}\left(\kappa^{+(\omega+1)},<j(\kappa)\right.$ ), $K=\operatorname{Coll}\left(\aleph_{0}, \kappa^{+\omega}\right)$, and then $P=Q \times K$ is the desired collapse. In $V^{P}, \kappa^{+\omega}$ is countable, $\kappa^{+(\omega+1)}$ is $\aleph_{1}, j(\kappa)$ is $\aleph_{2}$, and $\mu=\lambda^{+}=j(\kappa)^{+(\omega+1)}$ becomes $\aleph_{\omega}^{+}$. 
So let $\mathcal{S}_{0}$ be in $V^{P}$ any strong $\mu$-system; then $\operatorname{Pre}_{P}\left(\mathcal{S}_{0}\right)$ can be obtained in two stages, corresponding to the product $P=Q \times K$ and decomposition $V^{P}=\left(V^{Q}\right)^{K}$. First, in $V^{Q}$, form $\mathcal{S}_{1}=\operatorname{Pre}_{K}\left(\mathcal{S}_{0}\right)$. Then $\mathcal{S}_{1}$ is in $V^{Q}$ a strong system on $\mu$, with $|K| \times \aleph_{0}=\kappa^{+\omega}$ relations, one relation for each pair formed with a condition in $K$ and a relation (index) in $\mathcal{S}_{0}$. Hence, by the theorem, $\operatorname{Pre}_{Q}\left(\mathcal{S}_{1}\right) \sim \operatorname{Pre}_{P}\left(\mathcal{S}_{0}\right)$ has (in $V$ ) a narrow subsystem as required.

\section{The potential branching property and a model with no Aronszajn trees}

Suppose $\mu=\lambda^{+}$. The potential branching property for $\mu$ is the following statement:

If $\mathcal{S}$ is a narrow system on $\mu$, then for every $\chi<\lambda$ there is a $\chi$ complete forcing poset that introduces an unbounded branch to $\mathcal{S}$.

Recall that a branch of a system is a set of nodes and a relation in the system which includes every pair from the set. In the following section we will see how to obtain the potential branching property, but here we use it to obtain a model with no Aronszajn trees on $\aleph_{\omega+1}$.

Theorem 5.1 Let $\kappa$ be $\kappa^{+(\omega+1)}$-huge (as in Lemma 2.3). Suppose that the potential branching property for $\mu$ holds $\left(\mu=j(\kappa)^{+(\omega+1)}\right)$. Then there is a generic extension in which $\mu$ becomes $\aleph_{\omega}^{+}$and it carries no Aronszajn trees.

Proof. The poset $P$ of Corollary 4.2 works. Recall that $Q=\operatorname{Coll}\left(\kappa^{+(\omega+1)},<\right.$ $j(\kappa)), K=\operatorname{Coll}\left(\aleph_{0}, \kappa^{+\omega}\right)$, and then $P=Q \times K$ is the desired collapse. We will prove that there are no $\mu$-Aronszajn trees in $V^{P}$. The demonstration of this result depends on a preservation theorem which will only be proved in the following subsection, and it goes as follows.

Suppose that $\mathbf{T}$ is in $V^{P}$ a name of a $\mu$-tree. We will first show that there is in $V$ a $\kappa^{+(\omega+1)}$-complete poset $R$ such that, in $\left(V^{P}\right)^{R}$, $\mathbf{T}$ aquires an unbounded branch. Then the preservation theorem (5.2, applied with $\left.\lambda=j(\kappa)^{+\omega}\right)$ shows that $\mathbf{T}$ has a branch already in $V^{P}$.

To see how $R$ is obtained, apply the corollary to $\mathbf{T}$, considered as a one relation strong system, and find a narrow subsystem $\mathcal{S}$ to $\operatorname{Pre}_{P}(\mathbf{T})$. But then for $\chi=|P|<\mid$ lambda there is a $\chi^{+}$-complete forcing poset $R$ that introduces an unbounded branch to $\mathcal{S}$. This can be shown to give an unbounded branch to the tree $\mathbf{T}$ in $V^{P \times R}$. But then the following preservation theorem shows that $\mathbf{T}$ already has a branch in $V^{P}$.

\subsection{The preservation theorem}

Theorem 5.2 Let $\lambda$ be a singular cardinal (of cofinality $\omega$, for notational simplicity), and suppose that $P$ and $R$ are two posets such that: 
1. $\|P\|=\chi<\lambda$, and $\mathbf{T}$ is a $\lambda^{+}$-tree in $V^{P}$.

2. $R$ is $\chi^{+}-$closed.

Then any $\lambda^{+}$-branch of $\mathbf{T}$ in $V^{P \times R}$ is already in $V^{P}$.

Proof. As before, $T_{\alpha}=\lambda \times\{\alpha\}$ is the $\alpha$ th level of $\mathbf{T}$, for $\alpha<\mu=\lambda^{+}$. Let $\mathbf{B} \in V^{P \times R}$ be a name of a cofinal branch of $\mathbf{T}$, supposedly not in $V^{P}$. We also view $\mathbf{B}$ as a name in $\left(V^{P}\right)^{R}$ (that is, a name in $R$-forcing, in $V^{P}$ ).

Say (in $V^{P}$ ) that two conditions $r_{1}, r_{2} \in R$ force distinct values for $\mathbf{B} \cap T_{\alpha}$ iff for some $a_{1} \neq a_{2}$ in $T_{\alpha}, r_{i} \Vdash a_{i} \in \mathbf{B}$, for $i=1,2$. A weaker property, which even may hold when $r_{1}$ or $r_{2}$ do not determine $\mathbf{B} \cap T_{\alpha}$, is that whenever $r_{1}^{\prime}$ and $r_{2}^{\prime}$ are extensions of $r_{1}$ and $r_{2}$ that determine the value of $\mathbf{B} \cap T_{\alpha}$ then $r_{1}^{\prime}$ and $r_{2}^{\prime}$ force distinct values for $\mathbf{B} \cap T_{\alpha}$. In this case we say that $r_{1}$ and $r_{2}$ "force contradictory information on $\mathbf{B} \cap T_{\alpha}$ ". Observe that if $\alpha<\beta<\mu$ and $r_{1}, r_{2}$ force contradictory information on $\mathbf{B} \cap T_{\alpha}$, then they force contradictory information on $\mathbf{B} \cap T_{\beta}$.

Working in $V$, our aim is to tag the nodes of the tree $\lambda^{<\omega}$ (finite sequences from $\lambda$ ) with conditions in $R$ that are forced to force pairwise contradictory information on $\mathbf{B} \cap T_{\alpha}$ for some $\alpha$, and this will be shown to be impossible because the cardinality of $T_{\alpha}$ is $\lambda$ and $\lambda^{<\omega}$ has $\geq \lambda^{+}$branches. We will denote the tag of $\sigma \in \lambda^{<\omega}$ with $r_{\sigma} \in R$. The required properties of this tagging are the following.

1. If $\sigma_{1} \subset \sigma_{2}$ in $\lambda^{<\omega}$, then $r_{\sigma_{1}} \leq r_{\sigma_{2}}$ in $R$.

2. For every node $\sigma \in \lambda^{<\omega}$, for any two immediate extensions $\sigma_{1}, \sigma_{2}$ of $\sigma$, there are an ordinal $\alpha=\alpha\left(\sigma_{1}, \sigma_{2}\right)$, and a dense set $D \subseteq P$, such that for every $p \in D$

$$
p \Vdash_{P} \quad r_{\sigma_{1}} \text { and } r_{\sigma_{2}} \text { force contradictory information on } \mathbf{B} \cap T_{\alpha} .
$$

Why this suffices? Because, assuming such a construction, let $\beta<\mu$ be above all the ordinals $\alpha\left(\sigma_{1}, \sigma_{2}\right)$ mentioned in item 2 for nodes $\sigma_{1}$ and $\sigma_{2}$, and look at the set of all full branches $\lambda^{\omega}$. For each $f \in \lambda^{\omega}$, let $r_{f} \in R$ be an extension of the upper bound of the conditions $r_{f \nmid n}$, tagged along the branch $f$, an extension that determines the value of $\mathbf{B} \cap T_{\beta}$. We claim now that if $f \neq g$ are full branches, then there is a dense set $D \subseteq P$ such that for every $p \in D, p \Vdash_{P}$ $r_{f}$ and $r_{g}$ force distinct values for $\mathbf{B} \cap T_{\beta}$. Indeed, let $\sigma \subset f \cap g$ be the splitting node, then item 2 gives the required dense set. To conclude the proof, we find that, in $V^{P}$, any two branches of $\lambda^{\omega}$ give distinct values for $T_{\beta}$, and since $\lambda^{\aleph_{0}} \geq \lambda^{+}$, this shows that $T_{\beta}=\lambda \times\{\beta\}$ contains $\lambda^{+}$distinct nodes in $V^{P}$, which is not possible since $\lambda^{+}$is not collapsed.

In the construction of the tags, the following lemma, stated in general for any poset $S$, provides a basic tool. 
Lemma 5.3 Let $S$ be any forcing poset. Suppose that $\lambda$ and $\lambda^{+}$are a cardinal and its successor, $T$ is a $\lambda^{+}$-tree, and $\mathbf{B}$ is a name of a $\lambda^{+}$-branch of $T$ in $V^{S}$. If $\mathbf{B}$ is a new branch ( $\mathbf{B}$ is not in $V$ ), then for some $\alpha$ there is a set $X \subset T_{\alpha}$ of cardinality $\lambda$, in $V$, such that every $x \in X$ is forced by some condition in $S$ to be in $\mathbf{B}$.

Proof. We will say that $s \in S$ is $\lambda$-wide at $T_{\alpha}$ if there are $\lambda$ extensions of $s$ that force pairwise distinct values for $\mathbf{B} \cap T_{\alpha}$. If we start with an arbitrary condition, our proof will give that every $s \in S$ is $\lambda$-wide at some $T_{\alpha}, \alpha<\lambda^{+}$.

Define $E$, in $V$, to be the set of possible nodes of $\mathbf{B}$ :

$$
E=\{a \mid \text { some condition in } S \text { forces } a \in \mathbf{B}\} .
$$

We want some $\alpha<\lambda^{+}$such that $\left|E \cap T_{\alpha}\right|=\lambda$. So assume, on the contrary, that $\left|E \cap T_{\alpha}\right|<\lambda$ for every $\alpha$. Then $E \subseteq T$ satisfies the following properties:

1. Any node in $E$ has extensions in $E$ at arbitrarily higher level.

2. $E$ is downward closed in $T$.

3. Any node in $E$ has two incomparable extensions in $E$ (for otherwise, a condition would force that $\mathbf{B}$ is in $V$ ).

4. For every $\alpha<\lambda^{+},\left|E \cap T_{\alpha}\right|<\lambda$.

This is not possible: let $U \subseteq \lambda^{+}$be a closed unbounded set such that if $\alpha \in U$ then whenever $\gamma<\alpha$, if $a \in E \cap T_{\gamma}$, then $a$ has two incomparable extensions of height $<\alpha$ in $E$. Then pick any $\alpha \in U$ such that $\alpha \cap U$ has order-type $\geq \lambda$, and conclude that $E \cap T_{\alpha}$ has size $\lambda$ by splitting the points at levels in $U$ below a. QED

Returning to our case $S=P \times R$, we will say that $(p, r) \in P \times R$ is $\lambda$-wide at $T_{\alpha}$ if there are $\lambda$ extensions of $(p, r)$ that force pairwise distinct values for $\mathbf{B} \cap T_{\alpha}$. (This is not exactly the same definition as the one given above, because $\mathbf{T}$ is not assumed to be in $V$; however, its level-sets are, and so this definition is meaningful.)

Lemma 5.4 1. Any condition $(p, r) \in P \times R$ is $\lambda$-wide at some $T_{\alpha}$.

2. If $(p, r)$ is $\lambda$-wide at $T_{\alpha}$, then it is also $\lambda$-wide at any higher level $T_{\beta}$.

Proof. Indeed, let $G$ be a $V$-generic filter over $P$ containing $p$. In $V[G], \lambda$ and $\lambda^{+}$are not collapsed, and we can use Lemma 5.3 to find some $\alpha<\mu$ such that there are $\lambda$ possible values for $\mathbf{B} \cap T_{\alpha}$ (forced by some extensions of $r$ ). Any such value is also a possible value for some extension of $(p, r)$ (in $V$ ), and hence when the set of possible values for $\mathbf{B} \cap T_{\alpha}$ is calculated in $V$ it must have cardinality $\lambda$ as well.

For the second part assume that $(p, r)$ is $\lambda$ wide at $\alpha$. given any $\beta>\alpha$, find first $\left(p_{i}, r_{i}\right)$ extending $(p, r)$, for $i<\lambda$, that determine distinct values of 
$\mathbf{B} \cap T_{\alpha}$ and then extend each pair to a condition $\left(p_{i}^{\prime}, r_{i}^{\prime}\right)$ that determines $\mathbf{B} \cap T_{\beta}$. Even though it may be possible for two such extensions to determine the same point in $T_{\beta}=\lambda \times\{\beta\}$, it is not possible for $\|P\|^{+}$extensions to determine the same point (because in such a case we would have two extensions with compatible $P$ coordinates, and this is not possible). Hence, as $\|P\|<\lambda$, there are $\lambda$ possible values for $\mathbf{B} \cap T_{\beta}$. Observe, however, that if $(p, r)$ is $\lambda$-wide at $T_{\alpha}$, then extensions of $(p, r)$ need not be $\lambda$-wide at the same $T_{\alpha}$, and it may be necessary to go to higher levels.

Lemma 5.5 If $\left\{r_{j} \mid j<\lambda\right\} \subseteq R$, and $p_{0} \in P$ are given, then, for some ordinal $\alpha$, there are extensions $r_{j}^{\prime} \geq r_{j}$ in $R$, for $j<\lambda$, such that for every pair $i<j$ there is $p_{1} \geq p_{0}$ in $P$ such that $p_{1} \Vdash_{P} \quad r_{i}^{\prime}$ and $r_{j}^{\prime}$ force distinct values for $\mathbf{B} \cap$ $T_{\alpha}$.

Proof. First, by our last lemma, find for every $i<\lambda$ an ordinal $\alpha_{i}$ such that $\left(p_{0}, r_{i}\right)$ is $\lambda$-wide at $T_{\alpha_{i}}$, and then let $\alpha$ be above all of these $\alpha_{i}$ 's. By the second part of the lemma, each $\left(p_{0}, r_{i}\right)$ is $\lambda$-wide at $T_{\alpha}$. Now, by induction on $i<\lambda$, we will define an extension $r_{i}^{\prime} \geq r_{i}$, and two functions, $e_{i}$ and $f_{i}, e_{i}: P \rightarrow P$, and $f_{i}: P \rightarrow T_{\alpha}$, such that:

1. For every $a \in P, e_{i}(a)$ extends $a$, and $\left(e_{i}(a), r_{i}^{\prime}\right) \Vdash_{P \times R} \mathbf{B} \cap T_{\alpha}=\left\{f_{i}(a)\right\}$.

2. If $k<i<\lambda$ then

$$
f_{i}\left(p_{0}\right) \notin\left\{f_{k}(a) \mid a \in P\right\} .
$$

That is, the value of $\mathbf{B} \cap T_{\alpha}$ that $\left(e_{i}\left(p_{0}\right), r_{i}^{\prime}\right)$ determines is different from all the values determined by previous conditions.

Suppose that it is the turn of $r_{i}^{\prime}, e_{i}, f_{i}$ to be defined. Let $P=\{p(\xi) \mid \xi<\chi\}$ be an enumeration of $P$, starting with the given condition $p(0)=p_{0}$. By induction on $\xi<\chi$, we shall define a condition $r_{i}^{\xi} \in R$, and the values $e_{i}(p(\xi))>p(\xi)$, and $f_{i}(p(\xi)) \in T_{\alpha}$ such that:

1. $\left\langle r_{i}^{\xi} \in R \mid \xi<\chi\right\rangle$ form an increasing sequence of conditions extending $r_{i}$

2. $\left(e_{i}(p(\xi)), r_{i}^{\xi}\right) \Vdash \mathbf{B} \cap T_{\alpha}=\left\{f_{i}(p(\xi))\right\}$.

3. $f_{i}(p(0)) \notin\left\{f_{k}(a) \mid a \in P, k<i\right\}$.

First, use the fact that $\left(p_{0}, r_{i}\right)$ is $\lambda$-wide at $T_{\alpha}$ to find an extension $\left(e_{i}\left(p_{0}\right), r_{i}^{0}\right) \geq$ $\left(p_{0}, r_{i}\right)$ that forces $\mathbf{B} \cap T_{\alpha}=\left\{f_{i}\left(p_{0}\right)\right\}$ for a value $f_{i}\left(p_{o}\right)$ that satisfies (3) above. Then construct the increasing sequence $r_{i}^{\xi}$ and the values of $e_{i}$ and $f_{i}$ (using the $\chi^{+}$completeness of $R$ at limit stages), and finally define $r_{i}^{\prime}$ to be an upper bound in $R$ of that sequence.

Let us check that the requirements of the lemma are satisfied for $r_{i}^{\prime}$. If $k<i$ is any index, look at $p^{\prime}=e_{i}\left(p_{0}\right)$, and let $p_{1}=e_{k}\left(p^{\prime}\right)$. Then $p_{1}$ is as required, because the value of $\mathbf{B} \cap T_{\alpha}$ determined by $\left(p_{1}, r_{i}^{\prime}\right)$ (namely $f_{i}\left(p_{0}\right)$ ) is 
distinct from the one determined by $\left(p_{1}, r_{k}^{\prime}\right)$ (namely $f_{k}\left(p^{\prime}\right)$ ). This proves the lemma, and the following completes the proof of the theorem by showing how the tagging can be done.

Lemma 5.6 If $r \in R$, then there are extensions $r_{i}^{\prime} \geq r$ for $i<\lambda$ such that, for some $\alpha$, if $i<j<\lambda$ then for some dense set $D=D_{i, j} \subseteq P$, for every $p \in D$,

$p \Vdash_{P} r_{i}^{\prime}$ and $r_{j}^{\prime}$ force contradictory information on $B \cap T_{\alpha}$.

Proof. Enumerate $P=\{p(\xi) \mid \xi<\chi\}$. By induction on $\xi \leq \chi$ we define:

1. A sequence of conditions in $R,\left\langle r_{i}^{\xi} \mid i<\lambda\right\rangle$.

2. A family $D^{\xi}(i, j) \subset P$, increasing with $\xi$, for every $i<j<\lambda$. Finally, we will set $D(i, j)=D^{\chi}(i, j)$, and to ensure that $D(i, j)$ is dense we demand that $p(\xi)$ has an extension in $D^{\xi+1}(i, j)$.

3. An ordinal $\alpha(\xi)<\mu$.

We require that for each $i,\left\langle r_{i}^{\xi} \mid \xi<\chi\right\rangle$ forms an increasing sequence, beginning with $r_{i}^{0}=r$. (Finally, $r_{i}^{\prime}=r_{i}^{\chi}$ will be the required extension.)

At limit stages $\delta, r_{i}^{\delta}$ is an upper bound in $R$ of the conditions $r_{i}^{\xi}, \xi<\delta$. $D^{\delta}(i, j)$ is the union of $D^{\xi}(i, j)$ for $\xi<\delta$.

At successor stages, $\xi+1$, the extensions $\left\{r_{i}^{\xi+1} \mid i<\lambda\right\}$ are defined using the previous lemma for the collection $\left\{r_{i}^{\xi} \mid i<\lambda\right\}$ and the condition $p_{0}=p(\xi)$. The lemma gives an ordinal $\alpha=\alpha(\xi)$ and extensions $p_{1}(i, j) \geq p(\xi)$, such that

$$
p_{1}(i, j) \Vdash r_{i}^{\xi+1} \text { and } r_{j}^{\xi+1} \text { force distinct values for } \mathbf{B} \cap T_{\alpha} \text {. }
$$

Then we define $D^{\xi+1}(i, j)$ by $D^{\xi+1}(i, j)=D^{\xi}(i, j) \cup\left\{p_{1}(i, j)\right\}$. Finally, define $r_{i}^{\prime}=r_{i}^{\chi}, \alpha=\sup \{\alpha(\xi) \mid \xi<\chi\}$, and $D_{i, j}=D^{\chi}(i, j) . D_{i, j}$ is dense in $P$, because every $p(\xi)$ has some extension in $D^{\xi+1}(i, j)$.

\section{The final model}

For the consistency of no Aronszajn trees on $\aleph_{\omega+1}$ we must show how to obtain the assumptions of Theorem 5.1, namely how to get a cardinal $\kappa$ which is $\kappa^{+(\omega+1)}$-huge with the potential branching property for $j(\kappa)^{+(\omega+1)}$. We are going to do this in two stages: In the first, it is shown that whenever an $\omega$ sequence of supercompact cardinals conveging to $\lambda$ is collapsed, then the potential branching property for $\lambda^{+}$holds. In the second stage, this is combined with preparations described in Section 2.3 .

Theorem 6.1 Suppose $\left\langle\lambda_{i} \mid i<\omega\right\rangle$, with $\lambda=\bigcup_{i<\omega} \lambda_{i}$ and $\mu=\lambda^{+}$, is an increasing $\omega$-sequence of $\mu$-supercompact cardinals (except for $\lambda_{0}$ which is just a regular cardinal). Let $C=\operatorname{Coll}\left(\left\langle\lambda_{i} \mid i<\omega\right\rangle\right)$ be the full support iteration that makes $\lambda_{i}$ to be $\lambda_{0}^{+i}$. Then, in $V^{C}$, the potential branching property holds for $\mu$ : 
If $\mathcal{S}$ is a narrow system on $\mu$, then, for every $k<\omega$, there is a $\lambda_{k}$-complete forcing that introduces an unbounded branch to $\mathcal{S}$.

Proof. We will actually prove the following combinatorial statement in $V^{C}$ :

For every $n<\omega$ and function $F:[\mu]^{2} \rightarrow \chi$, where $\chi<\lambda_{n}$, there is a $\lambda_{n}$-complete forcing $C^{*}$ such that in $\left(V^{C}\right)^{C^{*}}$ the following holds: For some $\nu<\chi$ there is an unbounded set $U \subseteq \mu$ such that for every $\alpha_{1}<\alpha_{2}$ in $U$ there is $\beta>\alpha_{2}$ such that

$$
F\left(\alpha_{1}, \beta\right)=F\left(\alpha_{2}, \beta\right)=\nu .
$$

(We call such a set $U$ "a branch" of $F$.)

First, we shall see why this combinatorial statement suffices to prove the theorem. Let $\mathcal{S}=(T, R) \in V^{C}$ be a narrow system over $\mu$, and let $\chi<\lambda$ be such that the width of $\mathcal{S}$ and the cardinality of its index set are $\leq \chi$. Suppose that $\chi<\lambda_{n}$ and we will find a $\lambda_{n}$-complete forcing that introduces an unbounded branch to $\mathcal{S}$. For this, define in $V^{C}$ a function $F:[\mu]^{2} \rightarrow \chi^{3}$ by

$F\left(\alpha_{1}, \alpha_{2}\right)=\left(\zeta, \tau_{1}, \tau_{2}\right)$ iff the $\tau_{1}$ member of $T_{\alpha_{1}}$ and the $\tau_{2}$ member of $T_{\alpha_{2}}$ stand in the $\zeta$ relation $R_{\zeta}$.

Then, by the assumed combinatorial principle, there is an unbounded set $U \subseteq \mu$, and fixed ordinals $\nu=\left(\zeta, \tau_{1}, \tau_{2}\right)$ as in equation (2). This implies that the $\tau_{1}$-th points of $T_{\alpha}$ (that is $\left\langle\tau_{1}, \alpha\right\rangle$ ) for $\alpha \in U$ form an $R_{\zeta}$ branch of $\mathcal{S}$ (use item 2 in the definition of systems).

We now argue why it suffices to prove the combinatorial principle for $n=0$. Given $F:[\mu]^{2} \rightarrow \chi$ where $\chi<\lambda_{n}$ in $V^{C}$ (suppose for simplicity that every condition in $C$ forces that $F$ is into $\chi$ ), decompose $C \simeq C_{n} * C^{n}$ where $C_{n}=$ $\operatorname{Coll}\left(\left\langle\lambda_{i} \mid i \leq n\right\rangle\right)$, and $C^{n}$ is the name in $V^{C_{n}}$ of $\operatorname{Coll}\left(\left\langle\lambda_{i} \mid n \leq i<\omega\right\rangle\right)$. In $V^{C_{n}}$ define $\lambda_{m}^{\prime}=\lambda_{n+m}$. Then each $\lambda_{m}^{\prime}$, for $m>0$ is $\mu$-supercompact. (Indeed the embedding $j: V \rightarrow M$ with critical point $\lambda_{k}$, for $k>n$, can be extended in $V^{C_{n}}$ to an embedding of $V^{C_{n}}$ into $M^{C_{n}}$, where $M^{C_{n}}$ posseses the same $\mu$-closure properties.) Thus, if we know case $n=0$ of the theorem in $V_{0}=V\left[C_{n}\right]$, we could apply it there to $C^{n}=\operatorname{Coll}\left(\left\langle\lambda_{i}^{\prime} \mid i<\omega\right\rangle\right)$ and get in $V_{0}^{C^{n}}=V^{C}$ the desired $\lambda_{0}^{\prime}$-complete $\left(\lambda_{n}\right.$-complete) poset that adds a branch to $F$. To save ourselves from too many superscripts, we denote $V^{C_{n}}$ by $V$ and $M^{C_{n}}$ by $M$ and assume $n=0$.

So, returning to the theorem, assume that $G$ is a $V$-generic filter over $C$, and $F$ is in $V[G]$ a function from $[\mu]^{2}$ into $\chi<\lambda_{0}$. In the following lemma, we will describe a $\lambda_{0}$-complete forcing $P$ in $V[G]$ that introduces a $\mu$-branch to $F$. Let $j: V \rightarrow M$ be an elementary embedding with critical point $\lambda_{1}$, such that $j\left(\lambda_{1}\right)>\mu$ and $M$ is closed in $V$ under $\mu$-sequences. The following lemma will be proved later on. 
Lemma 6.2 There is in $V[G]$ a $\lambda_{0}$-complete poset $P$ such that in $V[G]^{P}$ there is an extension of the embedding $j$ to an elementary embedding of $V[G]$ into $N=M[j(G)]$.

Accepting the lemma for a moment $j(F) \in N$ can be defined; a function on $[j(\mu)]^{2}$ and into $\chi<\lambda_{0}\left(j\right.$ is the identity below $\left.\lambda_{1}\right)$.

Since $j(\mu)>j^{\prime \prime} \mu$, there is an ordinal $\beta<j(\mu)$ above all the ordinals in $j^{\prime \prime} \mu$. Now, for each $\alpha<\mu$ we can find some $\zeta<\chi$ such that

$$
j(F)(j(\alpha), \beta)=\zeta .
$$

Since $\chi<\lambda_{0}$, and as $\mu$ is regular in $V$ and no new sequences of length $<\lambda_{0}$ are added to $V$ in $V[G]^{P}$, we may find a single $\zeta$ such that for unboundedly many $\alpha$ 's the equality $j(F)(j(\alpha), \beta)=\zeta$ holds. Since $j$ is elementary, it follows that for any $\alpha_{1}<\alpha_{2}$ in this unbounded set $F\left(\alpha_{1}, b\right)=F\left(\alpha_{2}, b\right)=\zeta$ holds for some $b>\alpha_{2}$. Thus an unbounded $\mu$-branch for $\zeta$ was found in $V[G]^{P}$, which is a $\lambda_{0}$-closed extension of $V[G]$.

We turn now to the proof of Lemma 6.2. The collapsing poset $C_{1}=$ $\operatorname{Coll}\left(\lambda_{0},<\lambda_{1}\right)$ is a factor of $C=\operatorname{Coll}\left(\left\langle\lambda_{i} \mid i<\omega\right\rangle\right)$, and for simplicity of expression, we identify $c \in C_{1}$ with the condition $\left\langle c_{1} \mid i<\omega\right\rangle \in C$ defined by $c_{0}=c$ and $c_{i}=\emptyset$ for $i>0$.

Denote each $j\left(\lambda_{i}\right)$ with $\lambda_{i}^{*}$. Then $\lambda_{0}^{*}=\lambda_{0}$, but $\lambda_{1}^{*}>\mu$. In $M, j(C)$ is $\left[\operatorname{Coll}\left(\left\langle\lambda_{i}^{*} \mid i \in \omega\right\rangle\right)\right]^{M}$, and $C_{1}^{*}=\operatorname{Coll}\left(\lambda_{0},<\lambda_{1}^{*}\right)$ (which is the same- defined in $V$ or in $M)$, is a factor of $j(C)=\left[\operatorname{Coll}\left(\left\langle\lambda_{i}^{*} \mid i<\omega\right\rangle\right)\right]^{M}$.

Let $G \subseteq C$ be a $V$-generic filter over $C$. Observe that if $\left\langle c_{i} \mid i<\omega\right\rangle \in G$, then $c \in G$ as well. In order to extend $j$ on $V[G]$ and to prove the lemma, we should find in $V[G]$ a $\lambda_{0}$-complete poset $P$ such that in $V[G]^{P}$ there is a $V$-generic filter $G^{*}$ over $j(C)$ such that

$$
\text { If } g \in G \text {, then } j(G) \in G^{*} \text {. }
$$

If we do so, then an embedding of $V[G]$ into $M\left[G^{*}\right]$ can be defined as follows: For any $x \in V[G]$, let $\mathbf{x}$ be a name of $x$ in $V^{C}$. Then $j(\mathbf{x})$ is a name in $V^{j(C)}$ and we define $j^{\prime}(x)$ to be its interpretation in $V\left[H^{\prime}\right]$. We trust the reader to check that $j^{\prime}$ is a well defined elementary extension of $j$.

nstead of writing down $P$, we will describe an iteration of two extensions, each one $\lambda_{0}$-complete.

Since $\mu<\lambda_{1}^{*}$ is collapsed to $\lambda_{0}$ in $V^{C_{1}^{*}}$, Lemma 2.2 implies that there is a projection, $\Pi$, of $C_{1}^{*}$ onto $C$, which can be used to find a generic extension of $V[G]$ which has the form $V[H]$ for a $V$-generic filter $H$ over $C_{1}^{*}$ such that:

1. The passage from $V[G]$ to $V[H]$ is done by forcing with a $\lambda_{0}$-closed forcing.

2. For every $c \in C_{1}, c \in G$ iff $c \in H$. 
Thus, for every $g \in G, j(g) \in j(C) / H$. Indeed, any $g \in G$ has the form $g=\langle c, \bar{r}\rangle$ where $c \in C_{1} \cap G$, and $\bar{r}$ is the later part of the sequence. Then $j(g)=\langle c, j(\bar{r})\rangle$ where $c \in C_{1}^{*} \cap H$, and thus $j(g) \in j(C) / H$.

It follows that $j^{\prime \prime} G$ (the image of $G$ under the restriction of $j$ to $C$ ) is in $M[H]$ a pairwise compatible collection of conditions in $j(C) / H$. Since $j(C) / H$ is isomorphic to $\operatorname{Coll}\left(\left\langle\lambda_{i}^{*} \mid i \geq 1\right\rangle\right)$ in $M[H]$, it is $\lambda_{1}^{*}$-complete, and a supremum, denoted $s \in j(C) / H$ can be found for $j^{\prime \prime} G$. This is our "master condition": If $G^{*}$ is any $V[H]$-generic filter over $j(C) / H$, containing $s$, then:

1. $G^{*}$ is in fact $V$-generic over $j(C)$.

2. The forcing $j(C) / H$ is $\lambda_{1}^{*}$-complete in $M[H]$, and it is thence $\lambda_{0}$-complete in $V[H]$ (because $M[H]$ is $\lambda_{0}$-closed in $V[H]$ ).

\section{Conclusion}

We have proved the following theorem:

Theorem 7.1 Assume a cardinal $\kappa$ and sequence $L=\left\langle\lambda_{i} \mid i<\omega\right\rangle$ such that

$B_{1}:$ For $\lambda=\sup \left\{\lambda_{i} \mid i<\omega\right\}$ and $\mu=\lambda^{+}$, each $\lambda_{i}, i>0$, is $\mu$-supercompact.

$B_{2}: \kappa$ is the critical point of an embedding $j: V \longrightarrow M$ where $j(\kappa)=\lambda_{0}$ and $M^{\mu} \subseteq M$.

Then there is a generic extension in which there are no $\aleph_{\omega+1}$ Aronszajn trees.

Indeed, in Section 2.3 we saw that by making a preparatory extension we may assume that $\kappa$ is such that if $C=\operatorname{Coll}(L)$, then in $V^{C} \kappa$ is $\kappa^{+(\omega+1)}$-huge. So, we go to $V^{C}$, and find that the potential branching property for $\mu=j(\kappa)^{+(\omega+1)}$ holds (by Theorem 6.1). But now, in $V^{C}$, all the assumptions for theorem 5.1 hold. Thus, in a final extension, obtained as a product of $\operatorname{Coll}\left(\kappa^{+(\omega+1)},<j(\kappa)\right)$ and $\operatorname{Coll}\left(\aleph_{0}, \kappa^{+\omega}\right)$, there are no Aronszajn trees on $\aleph_{\omega+1}$.

\section{References}

Baumgartner, Iterated forcing (on reverse Easton)?

Jensen, Weak square $=$ special tree

Magidor, On reflecting stationary sets

Magidor Levinski Shelah, Chang's conjecture on $\aleph_{\omega}$

Schimmerling no weak square implies inner model with Woodin.

Solovay Strong axioms, GCH above supercompacts. 